\title{
Lifting degenerate neutrino masses, threshold corrections and maximal mixing
}

\author{
Wolfgang Gregor Hollik*† \\ Institut für Theoretische Teilchenphysik \\ Karlsruhe Institute of Technology \\ E-mail: wolfgang.hollikekit.edu
}

In the scenario with degenerate neutrino masses at tree-level, we show how threshold corrections with either non-trivial or trivial mixing at tree-level have the power to generate the observed deviations from a degenerate spectrum. Moreover, it is possible to also generate the mixing fully radiatively when there is trivial mixing at tree-level.

We give a brief overview over the topic and discuss the outcome of threshold corrections for degenerate neutrino masses in a supersymmetric model. A detailed description can be found in [1].

Flavorful Ways to New Physics - FWNP,

28-31 October 2014

Freudenstadt-Lauterbad, Germany

\footnotetext{
${ }^{*}$ Speaker.

${ }^{\dagger}$ Report number: TTP14-038
} 


\section{Introduction}

The origin of neutrino masses and mixings hints to new physics beyond the Standard Model. Measurements of neutrino oscillations in the last years completed our picture of neutrino flavour in a sense that now all the mixing angles are quite precisely measured. What is still unknown is the mass scale of the lightest neutrino, whereas the squared mass differences are known. The masses are then to be easily calculated:

$$
m_{1}=m_{0}, \quad m_{2}=\sqrt{m_{0}^{2}+\Delta m_{21}^{2}}, \quad m_{3}=\sqrt{m_{0}^{2}+\Delta m_{31}^{2}},
$$

where $\Delta m_{i j}^{2}=m_{i}^{2}-m_{j}^{2}$ and we constrained ourselves to a normal ordering in neutrino masses $\left(m_{3}>m_{2}>m_{1}\right)$. The precise values can be determined via a global fit on neutrino oscillation data [2]:

$$
\begin{aligned}
& \Delta m_{21}^{2}=7.50_{-0.17}^{+0.19} \times 10^{-5} \mathrm{eV}^{2} \\
& \Delta m_{31}^{2}=2.457 \pm 0.047 \times 10^{-3} \mathrm{eV}^{2} .
\end{aligned}
$$

In a world with $m_{0} \gg \Delta m^{2}$, we live in the quasi-degenerate scenario $m_{1} \approx m_{2} \approx m_{3}$. This situation immediately evolves as soon as a direct mass search experiment like KATRIN reports a positive result, which would be in this case $m_{0} \approx 0.35 \mathrm{eV}$ [3]. Cosmology also prefers degenerate neutrino masses, where the anticipated scale is a bit below the KATRIN limit [4] $\left(m_{0} \approx 0.1 \mathrm{eV}\right)$ and still compatible with the recent $95 \%$ upper limit on the sum of the three light neutrino masses obtained by the Planck collaboration [5] $\left(\sum m_{v}<0.24 \mathrm{eV}\right)$.

It is well known that quantum corrections become important for any type of quasi-degenerate spectrum-renormalization group (RG) corrections $[6,7,8,9,10,11,12,13]$ as well as low-energy threshold corrections $[14,15,16,17,18]$. In general, both contributions from the RG as well as the threshold corrections are to be added to the effective neutrino mass matrix and change the flavour alignment as long as they are not flavour universal:

$$
m_{A B}^{v}=m_{A B}^{(0)}+m_{A C}^{(0)} I_{C B}+I_{A C} m_{C B}^{(0)},
$$

where $I_{A B}=I_{A B}^{\mathrm{RG}}+I_{A B}^{\mathrm{th}}$ are the quantum corrections and $m_{A B}^{(0)}$ is the neutrino mass matrix at treelevel. Capital indices count interaction eigenstates, where in the following small indices represent the mass basis. By means of a unitary transformation $U^{(0)}$, we arrive at the unperturbed mass eigenbasis, where $m^{(0)}$ is diagonal

$$
m_{a b}^{v}=m_{a}^{(0)} \delta_{a b}+\left(m_{a}^{(0)}+m_{b}^{(0)}\right) I_{a b}
$$

and $I_{a b}=\sum_{A B} I_{A B} U_{A a}^{(0)} U_{B b}^{(0)}$. In the following, we focus on the influence of the threshold corrections and take only $I=I^{\text {th }}$.

\section{Degenerate masses and threshold corrections}

Eq. (1.4) let us appreciate the advantage of degenerate masses: either $m_{1}=m_{2}=m_{3}$ and the mixing at tree-level is trivial $\left(U^{(0)}=\mathbb{1}\right)$, or when the masses have opposite signs (e.g. $m_{1}=-m_{2}=$ $\left.m_{3}\right)$ the corrected mass matrix gets tremendously simplified. 
The case of Majorana neutrinos does not allow to rotate away as many CP phases as for Dirac fermions. In general, there are two more phases left, such that the complete diagonalisation matrix can be written as a product of a unitary matrix with three angles and one phase and a phase matrix: $U_{v}=U^{(0)} P$ with $P=\operatorname{diag}\left(e^{i \alpha_{1}}, e^{i \alpha_{2}}, 1\right)$. The Majorana phases $\alpha_{1,2}$ can then be absorbed in a redefinition of the masses instead of a redefinition of the fields:

$$
m^{(0)} \rightarrow P^{T} U^{(0) T} m^{(0)} U^{(0)} P=m_{0} \operatorname{diag}\left(e^{-2 i \alpha_{1}}, e^{-2 i \alpha_{2}}, 1\right) .
$$

We have chosen the phases in a particular way to have a real and positive $m_{3}$.

Under the assumption of CP conservation in the Majorana phases, we take $\alpha_{1,2} \in\left\{0, \pm \frac{\pi}{2}\right\}$ and get e.g. $m_{1}=-m_{2}=m_{3}$. Eq. (1.4) simplifies to

$$
m^{v}=m_{0}\left(\begin{array}{ccc}
1+2 U_{\alpha 1} U_{\beta 1} I_{\alpha \beta} & 0 & 2 U_{\alpha 1} U_{\beta 3} I_{\alpha \beta} \\
0 & -1-2 U_{\alpha 2} U_{\beta 2} I_{\alpha \beta} & 0 \\
2 U_{\alpha 1} U_{\beta 3} I_{\alpha \beta} & 0 & 1+2 U_{\alpha 3} U_{\beta 3} I_{\alpha \beta} .
\end{array}\right)
$$

The two-fold degeneracy reveals an initial mixing matrix at tree-level that can be whatever it may, generated by some underlying flavour symmetry. However, the two-fold degeneracy leaves one freedom of rotation in the plane of degeneracy, i.e. the 1-3 plane: $U^{(0)} \rightarrow U^{(0)} R_{13}$ that can be used to rotate away the off-diagonal entries in Eq. (2.1) by requiring

$$
\sum_{A B} U_{A 1}^{(0)} U_{B 3}^{(0)} I_{A B}=0
$$

Note that Majorana masses are symmetric $m_{A B}=m_{B A}$ as are the corrections $I_{A B}=I_{B A}$. It is generically difficult to accommodate for the present value of $\sin \theta_{13}$ and the two $\Delta m^{2}$ with a minimal set of flavour-diagonal threshold corrections. More details on an update of this scenario can be found in [1]. We now shall discuss the case where there is trivial mixing at tree-level and we generate the mixing genuinely by threshold effects.

The case of exact degeneracy is characterized by no mixing at the tree-level: mass and interaction eigenstates can be arbitrarily interchanged and the fields redefined. We have three free rotations. Therefore the observed non-trivial mixing has to be generated via the quantum corrections only:

$$
m^{v}=m_{0} \mathbb{1}+m_{0}\left(\begin{array}{lll}
I_{11} & I_{12} & I_{13} \\
I_{12} & I_{22} & I_{23} \\
I_{13} & I_{23} & I_{33}
\end{array}\right)
$$

which means that we need flavour off-diagonal corrections. How can we constrain those? The observed neutrino mixing angles allow to first work in some approximations and then see, whether small deviations from the approximation help to get a better fit to data. First of all, one mixing angle is close to maximal, $\theta_{23} \approx \frac{\pi}{2}$, which allows to perform the rotation in the 2-3 plane with $I_{33}=I_{22}$ and $I_{23}=I_{22}$ and we obtain

$$
I^{\prime}=U_{23}^{T} I U_{23}=\left(\begin{array}{ccc}
I_{11} & \frac{I_{12}+I_{13}}{\sqrt{2}} & -\frac{I_{12}-I_{13}}{\sqrt{2}} \\
\frac{I_{12}+I_{13}}{\sqrt{2}} & 2 I_{22} & 0 \\
-\frac{I_{12}-I_{13}}{\sqrt{2}} & 0 & 0
\end{array}\right),
$$


which, together with the observation that one mixing angle is small ${ }^{1} \theta_{13} \approx 0$ allows for the approximation $I_{13} \approx I_{12}$ and the last rotation is done with

$$
\theta_{12} \approx \frac{1}{2} \arctan \left(\frac{2 \sqrt{2} I_{12}}{2 I_{22}-I_{11}}\right) .
$$

In this way, there is one free mixing angle $\theta_{12}$ and the two $\Delta m^{2}$ to be determined by three corrections $I_{11}, I_{22}$ and $I_{12}$, which is good in the approximation but obviously not sufficient to fit all the data. The deviation of $\theta_{23}$ from $\frac{\pi}{2}$ can be obtained by a splitting $I_{33}=I_{22}+\varepsilon$ and $\theta_{13} \neq 0$ with $I_{13}=I_{12}+$ $\delta$. We then have five parameters $I_{11}, I_{12}, I_{22}, \varepsilon$ and $\delta$ to determine three mixing angles $\theta_{12}, \theta_{13}, \theta_{23}$ and two mass splittings $\Delta m_{21}^{2}$ and $\Delta m_{31}^{2}$.

$$
\begin{aligned}
& \left(\begin{array}{lll}
m_{0} & & \\
& \sqrt{m_{0}^{2}+\Delta m_{21}^{2}} & \\
& & \sqrt{m_{0}^{2}+\Delta m_{31}^{2}}
\end{array}\right)= \\
& m U\left(\theta_{12}, \theta_{13}, \theta_{23}\right)^{T}\left(\begin{array}{ccc}
1+I_{11} & I_{12} & I_{12}+\delta \\
I_{12} & 1+I_{22} & I_{22} \\
I_{12}+\delta & I_{22} & 1+I_{22}+\varepsilon
\end{array}\right) U\left(\theta_{12}, \theta_{13}, \theta_{23}\right),
\end{aligned}
$$

the degenerate mass parameter $m$ can be identified with $m_{0}$. Using the possible KATRIN discovery, $m_{0}=0.35 \mathrm{eV}$, we get

$$
I=\left(\begin{array}{ccc}
0.976 & 1.03 & 1.05 \\
1.03 & 4.75 & 4.75 \\
1.05 & 4.75 & 6.74
\end{array}\right) \times 10^{-3}
$$

\section{Threshold corrections in the $v$ MSSM}

The generic threshold corrections given in the previous section have the property of large flavour off-diagonal contributions. One viable model to produce such corrections is the Minimal Supersymmetric Standard Model with right-handed neutrinos ( $v$ MSSM) that generate small neutrino masses via a see-saw mechanism.

The superpotential of the model is given by

$$
\mathscr{W} \supset \mu H_{1} \cdot H_{2}+Y_{i j}^{v} H_{2} \cdot L_{L, i} N_{R, j}-Y_{i j}^{\ell} H_{1} \cdot L_{L, i} E_{R, j}+\frac{1}{2} M_{i j}^{R} N_{R, i} N_{R, j},
$$

and the soft breaking terms

$$
V_{\text {soft }}^{\tilde{v}}=\left(m_{\tilde{L}}^{2}\right)_{i j} \tilde{v}_{L, i}^{*} \tilde{v}_{L, j}+\left(m_{\tilde{R}}^{2}\right)_{i j} \tilde{v}_{R, i} \tilde{v}_{R, j}^{*}+\left(A_{i j}^{v} h_{2}^{0} \tilde{v}_{L, i} \tilde{v}_{R, j}^{*}+\left(B^{2}\right)_{i j} \tilde{v}_{R, i}^{*} \tilde{v}_{R, j}^{*}+\text { h. c. }\right),
$$

that have the power to introduce flavour-changing self-energies. We take the soft masses universal, $m_{\tilde{L}}^{2}=m_{\tilde{R}}^{2}=M_{\text {SUSY }} \mathbb{1}$, and determine values of $A_{i j}^{v}$ to give corrections like Eq. (2.7). The results are shown in Fig. 1, details on the calculation of threshold corrections in the VMSSM are given in [1].

\footnotetext{
${ }^{1}$ Recent data tell us rather $\theta_{13} \approx 9^{\circ}$. For the moment, we stick to $\theta_{13}=0^{\circ}$.
} 

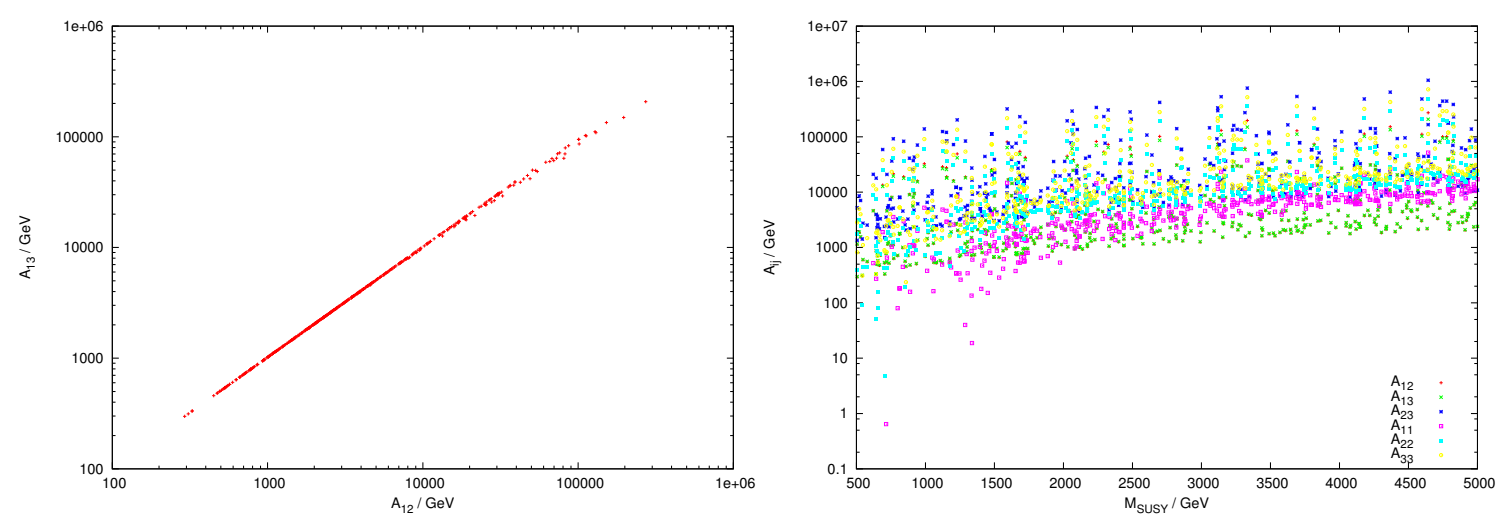

Figure 1: Results of the fit for a scan over several free parameters (details in [1]). The left plot shows the correlation between $A_{12}^{v}$ and $A_{13}^{v}$ which similar to the generic results of Sec. 2 . On the right side the typical size of $A_{i j}^{v}$ is shown with respect to the soft breaking mass $M_{\mathrm{SUSY}}$.

\section{Conclusions}

We have discussed degenerate neutrino masses at tree-level and investigated threshold corrections to two different mass patterns. In the scenario with one mass of a different sign, the minimal set of threshold corrections cannot produce the necessary deviation from degeneracy for the masses. On the other hand, generic threshold corrections can simultaneously generate all three mixing angles and the mass differences in the case of exactly degenerate masses and trivial mixing at tree-level. These findings have been applied to a supersymmetric model where the soft breaking terms carry the flavour information.

\section{Acknowledgements}

This work was supported by the GRK 1694 "Elementarteilchenphysik bei höchster Energie und höchster Präzision”. I appreciate valuable discussions with S. Pokorski about that topic. The workshop "Flavorful Ways to New Physics" was supported by the Karlsruhe House of Young Scientists (KHYS), the Karlsruhe School of Elementary Particle and Astroparticle Physics (KSETA), the KIT Center for Elementary Particle and Astroparticle Physics (KCETA) and the Graduiertenkolleg GRK 1694 as well as BlueYonder.

\section{References}

[1] W. G. Hollik, Radiative generation of neutrino mixing: degenerate masses and threshold corrections, arXiv: 1412.4585.

[2] M. C. Gonzalez-Garcia, M. Maltoni and T. Schwetz, Updated fit to three neutrino mixing: status of leptonic CP violation JHEP 1411 (2014) 052 [arXiv: 1409.5439 ].

[3] KATRIN Collaboration, A. Osipowicz et al., KATRIN: A Next generation tritium beta decay experiment with sub-eV sensitivity for the electron neutrino mass. Letter of intent, arXiv:hep-ex/0109033. 
[4] R. A. Battye and A. Moss, Evidence for Massive Neutrinos from Cosmic Microwave Background and Lensing Observations, Phys.Rev.Lett. 112 (2014) 051303 [arXiv: 1308.5870 ].

[5] Planck Collaboration, P. Ade et al., Planck 2013 results. XVI. Cosmological parameters, Astron.Astrophys. 571 (2014) A16 [arXiv:1303.5076].

[6] J. R. Ellis and S. Lola, Can neutrinos be degenerate in mass?, Phys.Lett. B458 (1999) 310-321, [arXiv:hep-ph/9904279].

[7] J. Casas, J. Espinosa, A. Ibarra, and I. Navarro, Naturalness of nearly degenerate neutrinos, Nucl.Phys. B556 (1999) 3-22, [arXiv: hep-ph/9904395].

[8] J. Casas, J. Espinosa, A. Ibarra, and I. Navarro, Nearly degenerate neutrinos, supersymmetry and radiative corrections, Nucl.Phys. B569 (2000) 82-106, [arXiv: hep-ph/9905381].

[9] N. Haba, Y. Matsui, N. Okamura, and M. Sugiura, The Effect of Majorana phase in degenerate neutrinos, Prog.Theor.Phys. 103 (2000) 145-150, [arXiv: hep-ph/9908429].

[10] K. Balaji, A. S. Dighe, R. Mohapatra, and M. Parida, Generation of large flavor mixing from radiative corrections, Phys.Rev.Lett. 84 (2000) 5034-5037, [arXiv: hep-ph/ 0001310 ].

[11] P. H. Chankowski and S. Pokorski, Quantum corrections to neutrino masses and mixing angles, Int.J.Mod.Phys. A17 (2002) 575-614 [arXiv: hep-ph/0110249].

[12] R. Mohapatra, M. Parida, and G. Rajasekaran, High scale mixing unification and large neutrino mixing angles, Phys.Rev. D69 (2004) 053007, [arXiv: hep-ph/ 0301234$]$

[13] N. Haba and R. Takahashi, Grand Unification of Flavor Mixings, Europhys. Lett. 100 (2012) 31001, [arXiv:1206.2793].

[14] E. J. Chun and S. Pokorski, Slepton flavor mixing and neutrino masses, Phys.Rev. D62 (2000) 053001, [arXiv: hep-ph/9912210].

[15] P. H. Chankowski, A. Ioannisian, S. Pokorski, and J. Valle, Neutrino unification, Phys.Rev.Lett. 86 (2001) 3488-3491, [arXiv: hep-ph/0011150].

[16] E. J. Chun, Lepton flavor violation and radiative neutrino masses, Phys.Lett. B505 (2001) 155-160, [arXiv:hep-ph/0101170].

[17] B. Brahmachari and E. J. Chun, Supersymmetric threshold corrections to $\Delta m_{\odot}^{2}$, Phys.Lett. $\mathbf{B 5 9 6}$ (2004) 184-190, [arXiv: hep-ph/0312030].

[18] R. Mohapatra, M. Parida, and G. Rajasekaran, Threshold effects on quasi-degenerate neutrinos with high-scale mixing unification, Phys.Rev. D71 (2005) 057301, [arXiv: hep-ph/0501275]. 